\title{
Análisis de controladores PID para una planta de segundo orden usando redes neuronales
}

\author{
César Naranjo ${ }^{1}$ \\ Universidad Internacional del Ecuador \\ ORCID: https://orcid.org/0000-0002-6494- \\ 5260 \\ cnestronic@gmail.com \\ Quito-Ecuador
}

\author{
Flor Romero ${ }^{2}$ \\ Universidad de Oriente \\ ORCID: https://orcid.org/0000-0001-8518- \\ 3923 \\ mariarosalesseli@gmail.com \\ Estado Sucre-Venezuela
}

Recibido (09/11/20), Aceptado (18/11/20)

\begin{abstract}
Resumen: Los sistemas de segundo orden son aquellos que responden a una ecuación diferencial de segundo orden. Se plantea el estudio de un sistema de segundo orden, dado por un circuito eléctrico RC. Se determinó la ecuación de transferencia y se analizó la respuesta transitoria empleando el método del Lugar Geométrico de las Raíces, así mismo se determinaron los coeficientes del controlador PID. A partir de la respuesta del controlador PID se creo y entreno una red neural backpropagation para su posterior sustitución del controlador PID clasico. En este trabajo se realiza el análisis de un caso de estudio de controlador PID donde se ha implementado una red neuronal artificial para evaluar la respuesta del mismo. Se observa que los resultados son aceptables y con un buena respuesta transitoria
\end{abstract}

Palabra Claves: Planta de segundo orden, MATLAB, Lugar Geométrico de las Raíces, Red Neural,

\section{PID controllers analysis For a second order floor using neuronal networks}

\begin{abstract}
Second order systems are those that respond to a second order differential equation. The study of a second order system is proposed, given by an RC electric circuit. The transfer equation was determined and the transient response was analyzed using the method of the Geometric Place of the Roots, likewise the coefficients of the PID controller were determined. From the response of the PID controller, a backpropagation neural network was created and trained to later replace the classic PID controller. In this work, the analysis of a PID controller case study is carried out where an artificial neural network has been implemented to evaluate its response. It is observed that the results are acceptable and with a good transient response.
\end{abstract}

Keywords: Second-order plant, MATLAB, Geometric Root Place, Neural Network, Backpropagation.

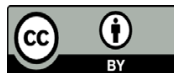




\section{I.INTRODUCCIÓN}

En ingeniería de control un sistema de segundo orden se caracteriza porque tiene dos polos. La función de transferencia genérica de un sistema de segundo orden en bucle cerrado tiene la forma de una ecuación cuadrática. Observando las raíces es posible encontrar un sistema subamortiguado, críticamente amortiguado o sobreamortiguado. En este artículo, se presenta un estudio experimental de un controlador PID basado en redes neuronales diseñado para un sistema de segundo orden. Se analiza el sistema dado por un circuito eléctrico de dos ramas. Se lleva a cabo la implementación de un controlador PID que permita la mejora del sistema. Empleando el método del Lugar Geométrico de las Raíces se determinaron los diferentes parámetros del controlador según los datos empleados para la planta del sistema. Se hace un estudio de la respuesta temporal del sistema y estos datos servirán para la implementación de la red neuronal. Posteriormente, se crea una red backpropagation y se entrena de modo que aprenda el comportamiento del controlador PID. Luego de entrenar la red, se introduce en el sistema de control y se hace un análisis de la respuesta transitoria con la red neuronal controlando el sistema.

En este trabajo se presenta un análisis del comportamiento de un caso de estudio donde se implementó un controlador PID a un circuito eléctrico RC. Para ello se analizó la ecuación característica y se validó el comportamiento utilizando la simulación en un software matemático.

Este trabajo consta de tres secciones, en la primera se han descrito de forma general los aspectos que contextualizan el trabajo, en la segunda sección se encontrarán los fundamentos teóricos de la investigación, luego se encontrará la metodología desarrollada y finalmente las resultados y conclusiones.

\section{II.DESARROLLO}

Un controlador PID es un mecanismo utilizado en los sistemas electrónicos de control, que está compuesto por tres partes fundamentales: Proporcional-Integral-Derivativo. Este permite calcular el error presente entre el valor medido y el valor deseado. Las principales aplicaciones se encuentran en sistemas industriales [1].

Los parámetros que caracterizan el controlador PID son el proporcional, que depende del error presente de forma actual, el integral que depende de los errores pasados, y el derivativo que es una predicción de los errores futuros. La combinación de estos tres elementos es utilizada para realizar los ajustes necesarios a través de algún elemento de control, que puede ser una válvula, una variable o un dispositivo electrónico de control.

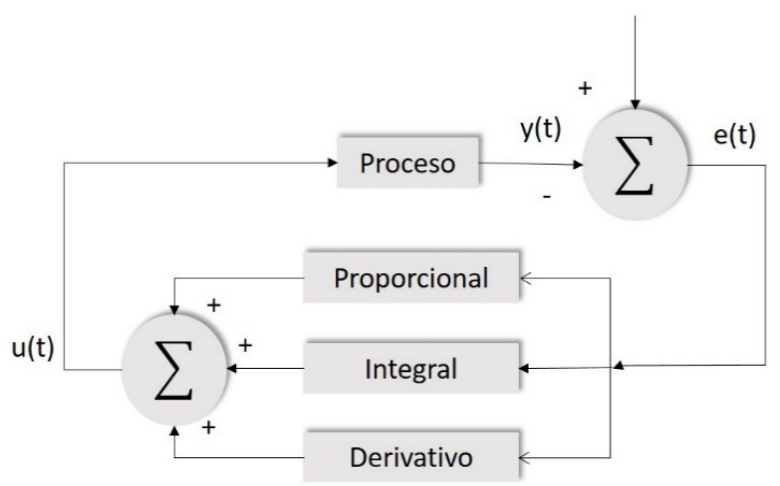

Figura 1. Elementos del controlador PID

El funcionamiento de un controlador PID estará sujeto a la combinación de otros elementos básicos que son el sensor, el controlador y el actuador. 


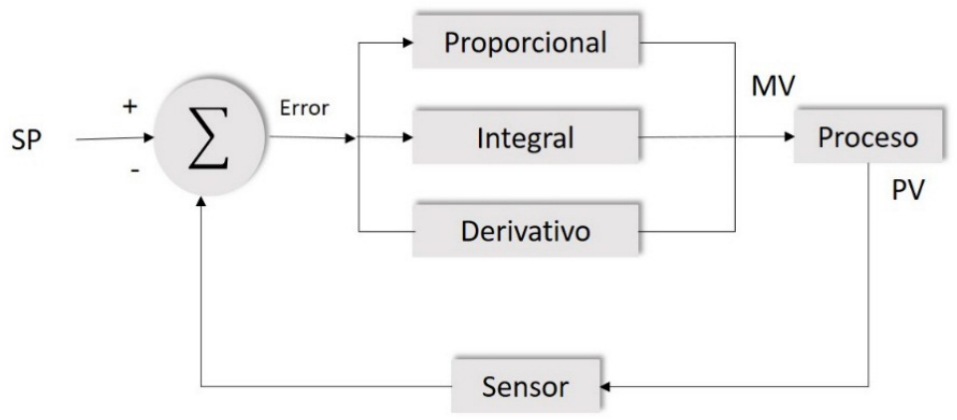

Figura 2. Elementos para el funcionamiento del controlador PID

El sensor es elemento que determina el estado del sistema, que permite capturar las variables de entrada que pueden ser calor, nivel, presión, entre otras. Por esto el sensor puede ser un termómetro, un caudalímetro, un termistor, un manómetro, una galga extensiométrica, entre muchos otros que puedan detectar variables de un proceso [2]. Este puede ser analógico o digital.

$\mathrm{El}$ actuador que pueda realizar las modificaciones necesarias para ejercer el control. Se encarga de restar la señal del nivel actual al valor de referencia, para dar el valor de error, que permite valorar la diferencia entre el valor deseado y el valor actual del sistema [3]. Esta señal es utilizada por el controlador para PID para gobernar y ejercer las acciones necesarias para mantener la estabilidad del sistema. La señal resultante de la suma de estas tres se llama variable manipulada y no se aplica directamente sobre el actuador, sino que debe ser transformada para ser compatible con el actuador utilizado [4], [5].

Finalmente el controlador se encarga de recibir la señal deseada, conocida como señal o punto de referencia. Esta señal es de la misma naturaleza y de iguales características que la señal que recoge el sensor, y será la necesaria para estimar el error [6].

\section{III.RESULTADOS}

\section{A.Un caso de análisis}

El circuito R-C mostrado en la figura 1, emula una planta de segundo orden. Se utilizará la función de transferencia de este circuito como planta para implementar un sistema de control a lazo cerrado digital, utilizando como controlador la tarjeta dsPIC de microchip.

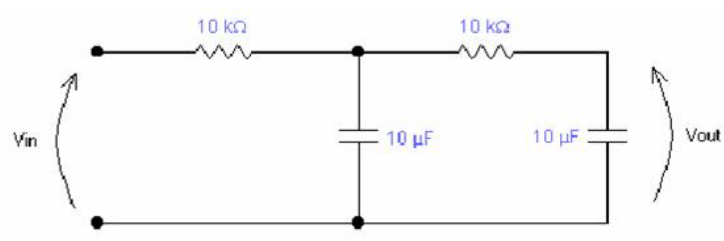

Figura 3. Circuito RC

Utilizando el software de programación matemática de este, se determinarán las características de la respuesta transitoria para un escalón unitario. Luego se diseñará un controlador PID continuo para este sistema de segundo orden, para satisfacer los siguientes requerimientos a lazo cerrado: $\square^{\wedge}{ }_{2}=0.707$ (Coeficiente de Amortiguamiento), fo $=0.5 \mathrm{~Hz}$ (Frecuencia Natural) Seguidamente se diseñará una red neuronal que cumpla las funciones del controlador. Por último se simulará el sistema continuo con controlador PID y red neuronal y se analizarán las respuestas temporales a fin de ver que efectos introduce en el sistema de control una red neuronal como elemento de control. 


\section{B.Análisis de la solución}

Para encontrar la solución idónea al problema propuesto, en primer lugar se llevó a cabo el análisis matemático del circuito propuesto.

1.- Del circuito de la figura 1, se obtienen las respectivas ecuaciones que determinan la ecuación de transferencia del sistema.

$$
G(S)=\frac{1}{0,01 S^{2}+0,3 S+1}
$$

Utilizando el software un programación matemático, se determinaron las características transitorias del sistema y una vez simulado éste se obtuvieron los siguientes parámetros de la respuesta temporal: $\% M_{p}=0 \%$

$t_{s}=0.46 \mathrm{seg} t_{r}=0.259 \mathrm{seg} t_{d}=0.123 \mathrm{seg}$

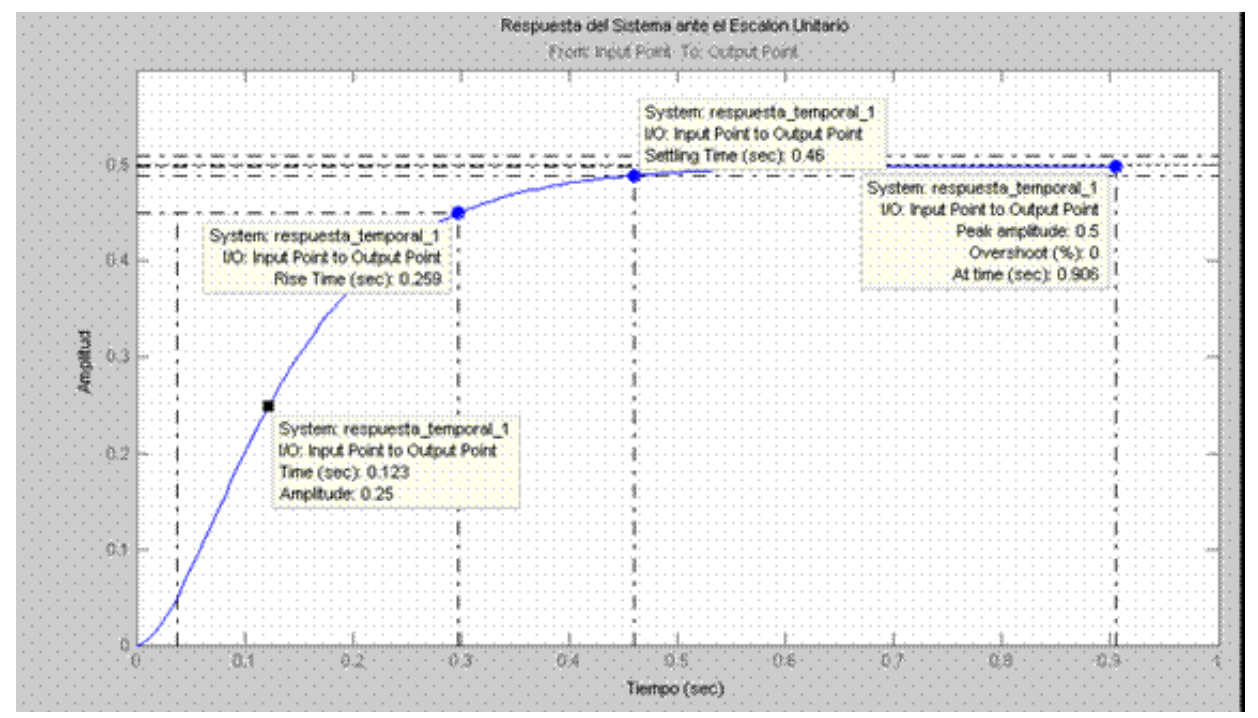

Figura 4. Respuesta del sistema con el controlador del método analítico

La figura 2 muestra la respuesta transitoria del sistema de segundo orden, en ella se puede observar que existe un tiempo de subida bastante lento y que dicha respuesta no alcanza el valor de estado estable en 1, donde se fijó el escalón unitario.

\section{C.Diseño del Controlador usando la Red Neuronal.}

Para este estudio se utilizó una red feedforward. Una de las características más importantes y poderosas de las redes neuronales feedforward es su capacidad como aproximadores universales, esto es, teniendo en cuenta cualquier función derivable y acotada, existe una red neuronal con un número suficiente de neuronas escondidas que puede aproximar dicha función con el nivel de precisión deseada. Es por ello que para este estudio se usa este tipo de red. El primer paso, es identificar los valores de entradas y salidas para la red. En este caso, los vectores de entrada y salida se toman de la señal de error del sistema de control (entrada del controlador) y la salida de control (salida del controlador). Se tomaron los datos obtenidos en el diseño matemático para comparar los resultados obtenidos utilizando solamente los datos que arroja el sistema de segundo orden. Una vez realizadas las pruebas se logró observar que ambos casos de estudios arrojaron resultados similares, siendo más exacto y preciso el controlador implementado con la red neuronal. La figura 3 muestra la respuesta del sistema con la red neuronal. En ella se observa que la respuesta es más rápida y que el tiempo de establecimiento es mucho menor. 


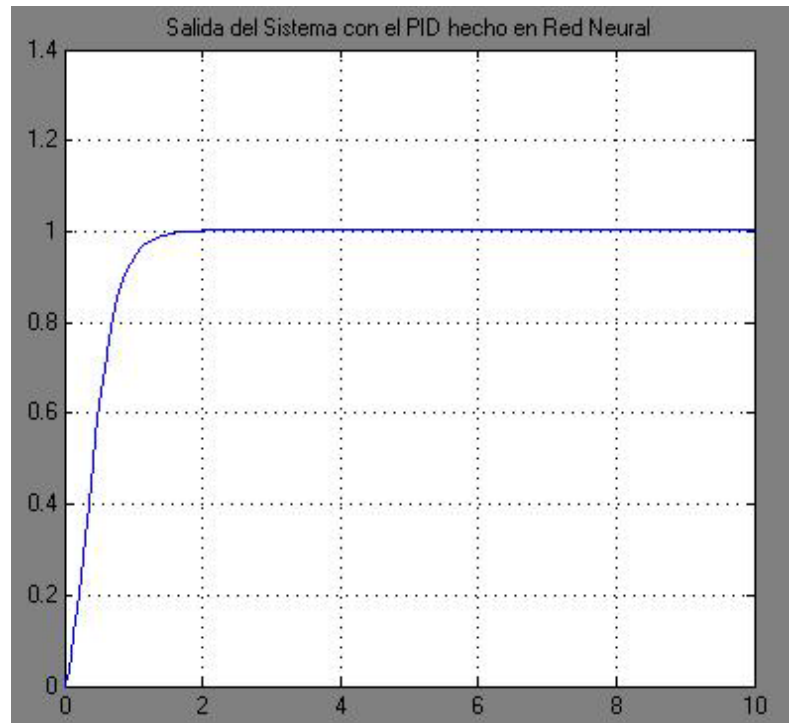

Figura 5. Respuesta del sistema con la red neuronal como algoritmo de control

\section{IV.CONCLUSIONES}

El diseño de controladores mediante el método analítico permite observar con detalles los ajustes en las ecuaciones relacionadas con el diseño. Sin embargo el diseño de controladores utilizando redes neuronales permite un mejor ajuste en el análisis del sistema sin necesidad de conocer las ecuaciones que lo rigen y por tanto ofrece una mayor precisión del comportamiento de las variables del proceso. El diseño de controladores auto ajustables con redes neuronales permite una respuesta rápida y efectiva del proceso.

\section{REFERENCIAS}

[1]Sistema de control en lazo cerrado. , «Sistema de control en lazo cerrado.,» [En línea]. Available: http:// www.des.udc.es/ luis/ControlIndustrial/CI.pdf. [Último acceso: 03 agosto 2008].

[Q]K. Astrom y T. HAgglum, «Revisando el método de respuesta escalonada de Ziegler-Nichols para el control PID,» Diario de control de procesos, vol. 14, nº 6, pp. 635-650, 2004.

[3]A. Hirata, L. Biagi, B. Angélico, P. Scalassara, W. Endo y R. Rodrigues, «implementação de um pid digital em ambiente computacional aplicado a uma planta didática para ensino de controle para engenharia,» de XLI Congreso Brasileiro de Educación en Ingeniería, Brasil, 2013.

[4]G. Kiam Heong Ang y Chong y Yun Li, «Análisis, diseño y tecnología del sistema de control PID,» IEEE Transactions on Control Systems Technology, vol. 13, no. 4, págs. 559-576, julio de 2005, doi:, vol. 13, $\mathrm{n}^{\circ}$ 4, pp. 559-576, 2005.

[5]K. Astrom y T. Hagglum, «El futuro del control PID,» vol. 9, $\mathrm{n}^{\mathrm{o}}$ 11, pp. 1163-1175, Control Engineering Practice.

[6]Sistemas de segundo orden. Referencia electrónica en línea. , «Sistemas de segundo orden. Referencia electrónica en línea.,» [En línea]. Available: http://es.wikipedia.org/wiki/Sistemas_de_segundo_orden. [Último acceso: 12 agosto 2008]. 